\title{
Relaciones especulares entre Marruecos y América Latina: de la generación Beat a la diplomacia cultural *
}

\author{
Sandra Rojo Flores ${ }^{* *}$
}

\section{Resumen}

Z $\mathrm{n}$ el presente texto, se exponen algunos ejemplos de las relaCiones especulares entre Marruecos y América Latina, privilegiando el caso de México. Se hace alusión a algunos fenómenos como la imagen de Marruecos y México en la mirada de la generación beat de los años cincuenta; Marruecos y América Latina durante los "años de plomo" marroquíes; la imagen de Marruecos como objeto de estudio y el imaginario marroquí sobre México. De la misma manera, se abordan algunos de los recientes proyectos e iniciativas que desplazan el sujeto-objeto hacia el sujeto-sujeto en el marco de los cada vez más numerosos proyectos comunes entre Marruecos y América Latina en el ámbito de la diplomacia cultural y las relaciones académicas, los cuales se han intensificado por el uso de las plataformas virtuales, debido a la crisis sanitaria ocasionada por el covid-19, lo que favorece la comunicación Sur-Sur.

\section{Palabras clave \\ Marruecos, mundo árabe-Latinoamérica, contracultura, cooperación Sur-Sur.}

Este trabajo fue realizado gracias al programa de Becas posdoctorales de la Coordinación de Humanidades de la UNAM, México.

" Instituto de Investigaciones Antropológicas. Universidad Nacional Autónoma de México. Contacto: srojo@ujaen.es ORCID: https:/orcid.org/0000-0001-9458-2467 
Fecha de recepción: abril de 2021
Fecha de aceptacion: junio de 2021

Specular Relations between Morocco and Latin America: from the Beat Generation to Cultural Diplomacy

\author{
Keywords \\ Morocco, Arab-Latin American world, counterculture, \\ South-South cooperation.
}

\title{
Abstract:
}

This article offers some examples of the specular relations between Morocco and Latin America, privileging the case of Mexico. The phenomena on which this paper focuses on are the image of Morocco and Mexico, in the gaze of the beat generation of the fifties; Morocco and Latin America during the Moroccan years oflead; the image of Morocco as an object of study, and the Moroccan imaginary about Mexico. At the same time, it aims to talk about some recent projects and initiatives, which shift the subject-object towards the subject-subject within the framework of the increasingly numerous common projects between Morocco and Latin America in the field of cultural diplomacy and academic relations. The latter is now more intense by using virtual platforms due to the health crisis caused by covid-19, favoring SouthSouth communication.

\section{Introducción}

En los últimos años, es notable el fortalecimiento de las relaciones académicas entre Marruecos y América Latina, fenómeno que se ha agudizado como consecuencia de la pandemia ocasionada por la covid-19. La posibilidad de llevar a cabo reuniones virtuales ha permitido superar la dificultad que implicaba el desplazamiento de los estudiantes e investigadores para asistir a cursos, coloquios o conferencias en ambas latitudes. El conocimiento de la lengua española por parte de un gran número de académicos marroquíes, fundamentalmente del norte del país y debido a las relaciones históricas de Marruecos con España, ha favorecido este 
intercambio, así como la existencia de departamentos de español en las diferentes universidades del país norteafricano. Estos nunca fueron ajenos a la producción literaria que se llevaba a cabo en Latinoamérica, y podemos incluso afirmar que el conocimiento de autores como Pablo Neruda, Mario Benedetti, Octavio Paz o Gabriel García Márquez formaron parte del corpus intelectual de muchos marroquíes, incluso fuera de esos ámbitos. Esto resulta evidente si se toma en cuenta que se trata de autores bastante conocidos, pero no deja de representar una ventaja sobre el conocimiento que de los autores y cultura marroquí se tiene en América Latina.

En las siguientes líneas, se hace un breve recorrido por algunas relaciones especulares entre Marruecos y América Latina, sobre todo en el ámbito cultural y académico, privilegiando el papel de México. Para ello, se considera preciso partir de una premisa principal: una especie de "horizontalidad natural" entre Marruecos y Latinoamérica que se puede interpretar desde al menos dos perspectivas. Ambas están presentes en las ideas del periodista y escritor español Rodolfo Gil Benumeya, representante del andalucismo africanista de los años veinte y treinta. La primera de ellas hace referencia a que, en diferentes momentos y con distintas duraciones, pasaron por una etapa de colonialismo.

Según Juan José Vagni, Gil Benumeya va más allá y “plantea una correspondencia entre ambos escenarios, donde las minorías criollas son asimiladas a la élite andalusí y los pueblos nativos americanos con las tribus bereberes" (Vagni, 2016, p. 41). Este discurso es puesto en marcha, según explica Vagni, con la intención de proporcionar un modelo americano para reforzar el Protectorado español en Marruecos y fortalecer el papel de España a nivel internacional "a través de su alianza con aquellos espacios regionales a los que está unida por historia y tradición: el mundo árabe y América Latina" (Vagni, 2016, p. 48).

El otro argumento esgrimido por Benumeya y vinculado con el anterior, es el relacionado con el "pasado andalusi', como escribe Vagni: "desde los años veinte, Gil Benumeya sostenía que tres ámbitos, el europeo (España), el árabe oriental y el hispano- 
americano aparecían intrínsecamente relacionados, fruto de la experiencia singular de al-Andalus y de las comunidades de emigración árabe en América” (Vagni, 2016-2017, p. 243).

Aunque los enfoques anteriores estuvieron dirigidos a crear un discurso que beneficiara a España y a la corriente africanista, es interesante constatar cómo ambos permanecen vigentes. En el primer caso, con el llamamiento a las relaciones Sur-Sur entre Marruecos y América Latina, como resultado de dos contextos similares señalados por una posición marginal en el contexto internacional, como ya lo detectara Gil Benumeya (Vagni, 2016-2017, p. 247). Así lo subrayó en una de sus alocuciones el exembajador de Marruecos en Colombia, Mohammed Ayachi en 1992: "Nosotros compartimos con estos países las mismas opciones para el arreglo de los problemas de descolonización, de lucha contra las secuelas de la colonización, de combate por el desarrollo económico y social, así como del estrechamiento de nuestras relaciones culturales" (Ayachi, 1992, p. 23).

En el segundo, por medio de la búsqueda de un pasado compartido cristalizado en un mito de lo andalusí, cuya connotación está invariablemente ligada a conceptos como la convivencia, la belleza o la sabiduría. Un mito que ha tenido diversas reinterpretaciones y que, tal como en la época de Benumeya, resulta útil para establecer una cercanía y fraternidad en el presente a través de una "herencia común". Hernán Taboada lo plasma con claridad cuando analiza la mirada del islam en América y considera que, en el caso del ensayo y heredada de Europa, "la veta que más ha confluido para dar una idea favorable al islam es la interpretación romántica de Al-Andalus como una isla de mestizaje, pluralidad cultural y emancipación femenina" (Taboada, 2003, p. 14).

En este sentido, es importante mencionar que a pesar de que se trata de un discurso que no solo abarca a Marruecos, sino que se extiende a Medio Oriente por las migraciones sirias y libanesas de finales del siglo XIX y principios del XX, el primero ocupa un lugar privilegiado en la construcción de dicha narrativa alimentado por la literatura, como es el caso en México del escritor Alberto Ruy Sánchez, quien ha llegado a acuñar el concepto de "orientalismo horizontal". 
Precisamente en este campo, el escritor chileno Sergio Macías, ha elaborado un concienzudo trabajo sobre la presencia de Marruecos en la literatura latinoamericana (Macías, 2014), así como lo ha hecho Hernán Taboada desde la historia al poner los cimientos del estudio de los viajeros mexicanos en Oriente, y lo que denomina un "orientalismo periférico" (Taboada, 1998). Entre ellos, incluye al general Ignacio Martínez Elizondo, médico y militar que emprendió sendos viajes alrededor del mundo en 1875 (Martínez, 1884) y 1883 (Martínez, 1886), visitando en el primero la ciudad de Tetuán, la que encontró desagradable, pues no puede olvidarse que se trataba de un momento político y social convulso, donde algunos abogaban por la modernización del país, como era el caso de Martínez Elizondo. Relata respecto a la ciudad marroquí:

[...] las calles estrechas y llenas de inmundicia, son intransitables por falta de luz y ventilación: los moros vestidos con babuchas de cordobán amarillo y sin medias, con una ancha capa blanca, amarillenta por la mugre, con un pantalón hasta la rodilla, la pierna desnuda y con un turbante o bonete, en su mayor parte parecen mendigos (Martínez, 1884, p. 261).

Son varias las dimensiones que pueden ser abordadas para retratar la relación entre Marruecos y América Latina, ya que además de la literaria y la histórica nos encontramos, entre otras, con la diplomática, la académica, la periodística y la popular, forjada sobre todo a través de las telenovelas, series y películas en ambos lados del Atlántico. Para acotar este trabajo, y con las premisas mencionadas anteriormente para establecer un ejercicio comparativo, se toma como referencia algunos aspectos para acercarnos a ciertos paralelismos tales como: a) México y Marruecos en la mirada de la generación Beat en los años cincuenta; b) algunas relaciones culturales y políticas entre América Latina y Marruecos vinculadas con los "años de plomo", periodo comprendido entre los inicios de la década de 1960 y finales de 1990;) la construcción de lo marroquí como objeto de estudio en México y la imagen de 
lo mexicano en el imaginario marroquí en las últimas décadas, $\mathrm{y}$ d) un acercamiento a algunos ejemplos de diplomacia cultural de Marruecos con México, Panamá, Chile y Argentina.

\section{México y Marruecos en la mirada de la generación Beat}

En los años cincuenta, momento en que algunos miembros de la generación Beat llegaron a Marruecos, habían pasado ya por algunos países latinoamericanos, entre ellos México. Tras su aventura mexicana, uno de sus máximos representantes, William Burroughs, quien había residido en la ciudad de México entre 1949 y 1952, se estableció en Tánger, concretamente entre los años 1953 y 1969.

Burroughs llegó a México acompañado de su esposa Joan Vollmer, huyendo de la justicia de su país. Un par de años más tarde, el asesinato accidental de Vollmer cuando jugaban a Guillermo Tell con un arma en una fiesta con sus amigos, le marcó para siempre. Entre su gente cercana no se encontraron nunca muchos mexicanos, salvo uno de sus biógrafos, el escritor Jorge García-Robles (García Robles, 2014 y 2018). Roger Bartra, al leer las memorias de la esposa de uno de los poetas beat, Bonnie Bremser, recuerda su forma de vida, prostituyéndose para mantener a su marido, lo que considera "una contradicción entre el espíritu liberador de los beats y la lamentable condición que ella vivía” (Bartra, 2017, \$ 15). Bartra narra cómo:

Los intelectuales de la contracultura veían a México solo como un espacio salvaje y atrasado y jamás se interesaron por sus manifestaciones intelectuales o políticas. Ni Ray Bremser, ni Allen Ginsberg ni Jack Kerouac se acercaron a los medios artísticos o intelectuales mexicanos. Tampoco Burroughs había tenido el menor contacto con la cultura mexicana. Al recordar aquella época, compruebo que en mi memoria hay muy pocas huellas del interés por México de los beats que pasaban por el país [...] (Bartra, 2017, §17). 
En el caso de Marruecos, Burroughs y otros escritores extranjeros de la época disfrutaron durante algunos años ese Tánger "internacional", como se le denominó a esta ciudad durante el Protectorado, pues estaba bajo el control de una comisión internacional a diferencia del resto del país que se encontraba bajo el mando de Francia o España. En ese paisaje político y cultural tildado de cosmopolitismo, Burroughs escribió una de las novelas más duras del mundo de las drogas: El almuerzo desnudo (Burroughs, 1989). El comportamiento de este y otros miembros de dicha generación con la población local, nos lleva a reflexionar sobre la semejanza entre la forma en que se integraron en ella, tanto en Marruecos como en México.

Juan Goytisolo describe muy bien esta relación en el caso del país norafricano, y no solo la de ellos, sino la de otros escritores como Paul y Jane Bowles, Francis Bacon, Truman Capote o Tennessee Williams. El escritor español afirma:

Como la totalidad de los escritores a los que antes me refiero, Burroughs se sentía a sus anchas en Tánger, pero no simpatizaba en exceso con los marroquíes. Si no se creía amenazado de muerte por estos, como Jack Kerouac durante las semanas que convivió con él, iba siempre armado bajo su proverbial impermeable oscuro y en razón de sus obsesivos vagabundeos por la Medina y la zona del puerto en busca de su dosis cotidiana de heroína era conocido como El hombre invisible de la famosa película por los asiduos del Zoco Chico (Goytisolo, 2014, \$4).

Como puede constatarse, la imagen romántica que se construye alrededor de estos, es puesta en cuestión por Goytisolo y lo es también por investigadores marroquíes como Anouar Majid, quien en el ciclo de conferencias organizado en 2014 en Tánger por la European Beat Studies Network sostuvo, refiriéndose al mismo grupo que: "La comunidad de occidentales prácticamente no salía de sus esferas particulares, nunca aprendieron la lengua y utilizaron la ciudad como escenario exótico de sus preocupaciones del primer mundo" (Mendoza, 2014, \$7). 
Además de la poca empatía hacia la población local, se encuentra un paralelismo en el caso de Marruecos y México en particular, y Latinoamérica en general, respecto a la búsqueda de ciertas drogas como en yage o ayahuasca en el caso de Sudamérica, y el majoun en el de Marruecos, además de la impresión de poder hacer, en ambos países, prácticamente lo que deseaban. Goytisolo reproduce las palabras de Burroughs al referirse a Tánger, quien consideraba que:

Es uno de los pocos lugares que aún quedan en el mundo donde en la medida que no cometes un atraco, empleas la violencia ni asumes abiertamente una conducta antisocial puedes hacer exactamente lo que quieres. Es el santuario de la No Interferencia (Goytisolo, 2014, §4).

Para Daniel Hiernaux-Nicola, la visión de México de otro beat, Jack Kerouac, era del "Disneylandia de los beat". Citando a García- Robles, recuerda como Burroughs "No demostró mucho interés ni siquiera para conocer mejor los barrios donde vivió, a pesar de la vida cultural y social significativa que en ellos se presentaba a fines de los cuarenta e inicios de los cincuenta" (Hiernaux-Nicola, 2007, p. 36). Tanto para Kerouac como para Burroughs, se trataba de un espacio de "liberación”, aunque más tarde también lo fuera de desesperanza, como se puede leer en el famoso libro de Jack Kerouac, Tristessa (Kerouac, 1960).

Burroughs refleja claramente lo anterior en su libro Queer escrito entre 1951 y 1953 en la Ciudad de México (Burroughs, 1985), traducido posteriormente en una de sus ediciones como Marica (Burroughs, 2006). El autor estadounidense nos introduce a la historia contando cómo:

En 1949 (México) era un lugar barato para vivir, con una enorme colonia extranjera, famosos burdeles y restaurantes, peleas de gallos y corridas de toros y cualquier forma imaginable de diversión. Un hombre podía vivir bien allí por dos dólares diarios [...] México era fundamentalmente una cultura oriental que 
reflejaba dos mil años de enfermedad y pobreza y degradación y estupidez y esclavitud y brutalidad y terrorismo psíquico y físico. Era siniestro y sombrío y caótico, con el caos especial de un sueño [...] (Burroughs, 2006, p.19).

Se tiene, pues, dos contextos donde se puede encontrar un denominador común que permitiría afirmar que ambos países poseen una característica que sirvió de fuente de inspiración y de telón de fondo presente en toda vida y obra de la generación beat: la miseria. Esta no solo es concebida por estos como una falta de recursos económicos, sino, sobre todo, como una serie de comportamientos que producían en ellos una suerte de atracción repulsión ante los lugareños que se cruzaron por sus caminos-. Lo anterior se percibe en sus escritos y actitudes, que se pueden encuadrar bajo el concepto de nostalgie de la boue (nostalgia por el fango), que fue utilizada a mediados del siglo XIX en Francia por el dramaturgo francés Émile Augier para describir a los burgueses atraídos por los bajos fondos.

No es casual que uno de los espíritus más potentes de dicha generación presente en los escritos de los demás, pero sin haber escrito sus propias páginas, Neal Cassady proviniera de una base social humilde, muy distinta a la de los otros. Muerto en 1968 al lado de las vías del ferrocarril, Charles Bukowski, en Escritos de un viejo indecente deja esta cruda imagen:

Tantos viajes, tantas páginas de Kerouac, tanta cárcel, para morir solo bajo una gélida luna mexicana, solo, ¿comprendes? ¿ves los pequeños cactus miserables? México no es un sitio malo simplemente porque esté oprimido; México es un mal sitio simplemente, ¿ves cómo miran los animales del desierto? las ranas, cornudas y simples, esas serpientes como hendiduras de mentes humanas que reptan, se paran, esperan, mudas bajo una muda luna mexicana, reptiles, rumores de cosas, contemplando a aquel tipo allí en la arena con su camiseta blanca de manga corta (Bukowski, 1998, p. 15). 
En la imagen que se acaba de exponer, tanto Marruecos como México, son considerados lugares donde la imaginación de un grupo de intelectuales, en este caso estadounidenses, gozaba de rienda suelta gracias a las condiciones de miseria y libertad que estos encontraban en ambos países. Este contexto se encuentra muy bien reflejado, en el caso de México, en la célebre película de Luis Buñuel Los olvidados, filmada en marzo de 1950 o en el libro del antropólogo estadounidense Oscar Lewis Los hijos de Sánchez (Lewis, 1965).

En el caso de Marruecos, los desgarradores escritos del escritor rifeño Mohamed Choukri como Elpan a secas, traducido inicialmente al español como El pan desnudo y posteriormente como $E l$ pan a secas (Choukri, 2014), no solo dan cuenta del ambiente del que se sirvieron varios escritores de ese grupo para sus escritos, sino de la relación abusiva o racista hacia ellos y la población local. Es el caso de Paul Bowles, escritor también estadounidense quien vivió cuatro años en México en los años treinta y en Marruecos desde 1947 hasta su muerte en 1999, cercano al grupo de Burroughs. Tanto Choukri (Choukri, 2012), como el también escritor Mohamed Mrabet, le dedican palabras muy duras. Se termina este apartado con las declaraciones de este último, quien estuvo al lado de Bowles durante 45 años y declaró en 2019 a la prensa cómo:

Llegaban cheques de hasta 300 dólares, pero al principio no le decía nada a su mujer. Luego ella le dijo que me diera algo, pero nunca recibí ni un franco. Dejé en un armario de ellos piezas de oro y plata de hace 100, 200 años, pinturas mías, todo se fue. En Nueva York se hicieron exposiciones con los libros de mis historias. Todos sus libros están hechos con mis historias. Digo la verdad, lo juro por dios, por mis hijos y mis nietos, era un hijo de la gran puta. Nunca les hice daño, le ayudé cuando estaba enfermo, le afeitaba, le llevaba en la cama. Porque el Corán dice que hay que ayudar a los judíos, a los budistas, a todos (Llorente, $2019, \$ 4)$. 
Algunas relaciones culturales y políticas entre Marruecos y América Latina vinculadas con los "años de plomo"

En el ámbito global latinoamericano, a finales de los años cincuenta, período de la Independencia de Marruecos, existía un ambiente permeado por una serie de dictaduras como la de Rafael Leónidas Trujillo en República Dominicana (1930-1961), Fulgencio Batista en Cuba (1952-1959), la familia Somoza en Nicaragua (1934-1979), Carlos Castillo Armas en Guatemala (1954-1957) o Alfredo Stroessner (1954-1989) en Paraguay.

El país magrebí obtuvo la independencia de España y Marruecos en 1956, dando fin al régimen del Protectorado que había estado vigente durante 44 años. Sin embargo, el clima posindependentista fue bastante traumático. Tras el retorno del sultán Mohamed V, exiliado por las autoridades francesas (1953-1956) y después de su muerte, se desencadenaron los llamados "años de plomo" (1961-1999) bajo el régimen de su hijo, Hassan II. Estuvieron caracterizados por la represión, encarcelación, desaparición y exilio de una gran cantidad de militantes políticos disidentes del sistema. Este periodo coincide con otro grupo de dictaduras latinoamericanas que iniciaron en los años setenta, como es el caso de Hugo Banzer en Bolivia (1971-1978), Jorge Videla en Argentina (1976-1981) o Augusto Pinochet en Chile (1973-1990).

Fue un momento en el que surgieron en el país africano varios movimientos contraculturales, tanto en el campo de la literatura como de las artes plásticas, el teatro, el cine o la música, entre otras expresiones. Asimismo, al término de esta época, y con el interés de gestionar las fracturas ocasionadas por dicha convulsión social y política, se consolidaron varias organizaciones de derechos humanos con la intención de encontrar modelos de gestión de la memoria, en este caso traumática, y algunos de ellos propusieron inspirarse en casos como los de Argentina o Chile para llevar a cabo su propio proceso de reconstrucción nacional, como el Centro para la Memoria Común, la Democracia y la Paz.

En el caso de la literatura, es imprescindible mencionar la aparición de la revista Souffles en 1966, fundada por los más desta- 
cados intelectuales y artistas de izquierda, entre ellos el conocido poeta fesí Abdelatif Laâbi, encarcelado de 1972 a 1980. Esta publicación representó todo un movimiento intelectual y político que fue prácticamente desconocido o poco estudiado no solo en el ámbito hispanófono, sino también en el propio Marruecos hasta la aparición, en el primer caso, del trabajo de la poeta Laura Casielles (Casielles, 2018) y, en el segundo, unos años antes, de Kenza Sefrioui (Sefrioui, 2013).

Otras revistas no menos importantes fueron Lamalif o $A l$ Asas, aunque no han corrido la misma suerte que la primera respecto a su recuperación por las actuales generaciones. Asimismo, se formó un interesante corpus de literatura carcelaria, como es el caso de la novela de Jaouad Mdidech La chambre noir (Mdidech, 2002), llevada al cine por Hassan Benjelloun en el año 2004; Tazmamart Cellule 1010 de Ahmed Merzouki (Merzouki, 2000); Le marié. Candide au pays de la torture del poeta Salah el Ouadie (Ouadi, 2001), así como la extensa obra de Laâbi o Abraham Sefarty entre otros, recogidos en los trabajos del investigador marroquí Khalid Zekri (Zekri, 2011).

Respecto a las artes plásticas es imprescindible mencionar al grupo de artistas que, reunidos en la conocida plaza Jemaa el Fna, firmaron el "Manifiesto de Marrakech" en 1965, coronado por una exposición colectiva. Fue, en sus palabras, la primera "fuera del marco de las misiones extranjeras" (Maraini, 1990, p. 114). Entre estos artistas destacan el recién desaparecido pintor Mohamed Melehi, así como Farid Belkahia o Mohammed Chebaa, por mencionar los más representativos, quienes formaron además parte activa de la revista Souffles.

El cine merece una mención aparte, ya que además de que Marruecos dejó de ser un país meramente decorativo para películas extranjeras, un sector fue conformando su propio estilo y carácter reivindicativo cercano a América Latina. Es el caso de la aparición, en 1970, de la revista Cinema 3, dirigida por Noureddine Sail y que hacía referencia a la idea de un "tercer cine”. Esta es una de las vías de conexión más representativas durante este período, ya que la idea de Sail aludía abiertamente a la propuesta, en esa 
época, de los directores argentinos Pino Solanas y Octavio Guettino de un cine contrapuesto al modelo comercial e imperialista de la época. En esta misma línea, encontramos el trabajo del director, originario de la ciudad de Oujda y colega de Sail, Mostafá Derkaoui, quien en su filme De quelques événements sans signifcation (Acerca de algunos eventos sin sentido), filmado en el año 1975 declara, a través de uno de los personajes que:

El cine marroquí debe ser comprometido y centrarse en la clase obrera. No es solo una cuestión marroquí sino del Tercer Mundo. Lo vemos en algunas películas de jóvenes cineastas latinoamericanos. No quiero que el cine de aquí se parezca al egipcio (Derkaoui, 1975)

Ahora bien, en el campo de la música, es imperativo hablar de grupos como Nass al Ghiwane, Jil Jilala o Lemchaheb, todos ellos cantantes en dariya, es decir, árabe marroquí, cuya estética y letras no están nada lejanos de grupos latinoamericanos Inti Illimani o Quilapayun, los cuales no les eran desconocidos. Estos grupos, sobre todo Nass al Ghiwane a través de su líder Larbi Batma, estaban muy ligados a uno de los hombres de teatro más reconocidos del medio marroquí: Tayeb Saddiki. La música de Nass al Ghiwane fue llevada al cine por el director Ahmed el Maânouni en el documental Transes (1981).

Las noticias que llegaban desde Latinoamérica estaban además trianguladas por la residencia temporal de muchos de estos artistas en países de Europa, entre ellos Francia, donde los movimientos de izquierda latinoamericanos no eran desconocidos y en el que era habitual escuchar a cantantes como la estadounidense Joan Baez, a través de la cual se escuchaban canciones de protesta de países como Chile.

Llegados a este punto, puede decirse que, mientras el mundo latinoamericano no era del todo ajeno a los movimientos de reivindicación artística y política de Marruecos, no sucedía lo mismo en el caso contrario, donde Marruecos aún se confundía, en el mejor de los casos, entre la nebulosa del árabe y no poseía 
una identidad propia. Los acercamientos en el mundo del cine o de la música de los realizadores y artistas marroquíes hacia Latinoamérica, no eran el resultado de una influencia gratuita de Latinoamérica hacia Marruecos, sino el reconocimiento de una estructura social similar donde ambas sociedades eran víctimas del colonialismo e imperialismo tanto económico como cultural, en la línea de lo que hemos mencionado en la introducción de este trabajo.

Ahora bien, muchos de estos intelectuales y artistas habían sido sujetos del régimen represivo y lo hicieron constar en sus obras y memorias, pero también se crearon mecanismos estatales y de la sociedad civil al término de dicho período para gestionar las fracturas sociales producidas durante esos años en el grueso de la población.

De parte del gobierno y coincidiendo con el final del reinado de Hassan II, se instauró el Foro Verdad y Justicia (1999), y más tarde la Instancia para la Equidad y Reconciliación (2004-2005), ya con el actual rey Mohamed VI. Esta última fue la encargada de llevar a cabo los procesos de reparación económica hacia las víctimas con el fin de llegar a un nuevo pacto social.

Este procedimiento de reconciliación se enlazó con América Latina, específicamente con Chile y Argentina, es decir, en la Comisión Nacional de Verdad y Reconciliación establecida por el presidente chileno Patricio Aylwin en 1990 y la Comisión Nacional sobre la Desaparición de Personas creada por el presidente argentino Raúl Alfonsin en 1983, destinadas a esclarecer la verdad sobre las violaciones a los derechos humanos entre 1973 y 1990 (Chile) y entre 1976 y 1983 (Argentina).

Algunos de los integrantes del IER marroquí, buscaron referentes en sus procesos, concretamente en la llamada justicia transicional. Sin embargo, y a pesar de las similitudes, existió siempre una característica importante para comprender la especificidad del caso marroquí. En el ejemplo latinoamericano, la transición se acompañó de la ruptura de un régimen anterior por uno nuevo, en el caso de Marruecos, esta transición se llevó a cabo dentro del mismo régimen, lo que condicionó absolutamente su devenir. Por 
ello, aunque estructuralmente se trató de situaciones similares, es decir un período de tiempo bajo una dictadura donde una parte de la población opuesta al régimen oficial sufrió desapariciones forzosas, asesinatos, encarcelamientos, etcétera, el procedimiento difería.

En el caso marroquí, se evitó nombrar a los culpables y fincar responsabilidades, asumiendo dicho proceso como una reconciliación necesaria entre partes igualmente responsables. Es importante advertir que este proceso se encuentra aún inconcluso sobre todo las regiones históricamente marginadas como sucede en la zona del Rif, tal como lo demuestra la investigación del realizador marroquí Tarik el Idrissi Rif 58-69 Briser le silence (Idrissi, 2014).

La Construcción de lo marroquí como objeto de estudio en México y la imagen de lo mexicano en el imaginario marroquí en las últimas décadas

Tanto la cuestión de los movimientos contraculturales y de vanguardia de los años sesenta y setenta de Marruecos, así como del acercamiento a América Latina a través de estos o de la búsqueda de similitud en el caso de los procesos de reconciliación nacional, no fueron nunca objeto de estudio en el ámbito latinoamericano, entre ellos el mexicano.

Puede afirmarse que el mundo árabe en general y magrebí en particular - asumiendo que dentro de este último existe una pluralidad étnica y lingüística importante que no se puede reducir a lo árabe - fue en un primer momento un objeto de estudio privilegiado casi exclusivamente para los antropólogos, debido a su formación académica al respecto. Sin embargo, el foco no se centraba en aspectos contemporáneos como los que acabo de mencionar, pues aún se consideraba a algunos países, entre ellos Marruecos, con un carácter arcaico donde manifestaciones como estas no tendrían cabida. No se concebía, pues, una región con una vanguardia artística y literaria que mirara hacia Latinoamérica para buscar inspiración en la música, el cine o la política. El Marruecos que se esbozaba era, básicamente, heredado de la mi- 
rada colonialista francesa o estadounidense, centrada en lo rural o en lo tribal y en las expresiones culturales expresamente chocantes para Occidente.

De esta forma, el currículo incluía los estudios de Clifford Geertz, en cuya obra aparece Marruecos como parte del corpus etnográfico de su antropología interpretativa (Geertz, 1994); de Paul Rabinow que ofrece su experiencia en el Atlas medio marroquí como material precioso para la reflexión sobre las vicisitudes del trabajo de campo (Robinow, 1974); de Ernest Gellner — conocido por su obra ineludible sobre el nacionalismo- quien dedicara parte de su investigación antropológica a la figura de la zaouias (cofradía) marroquí en el Atlas -y dentro de esta a la del Santo (Gellner, 1969) — o de David Montgomery Hart en el caso concreto del Rif marroquí (Hart, 1976). Muchos fueron los textos básicos en la formación antropológica durante generaciones, de la misma manera que algunos conceptos se instalaron en nuestro vocabulario académico, incluso más allá de la antropología, como el de "despotismo oriental" acuñado por el dramaturgo e historiador germano estadounidense K. A. Wittfogel (Wittfogel,1966).

Durante muchos años este interés fue meramente teórico debido, como se ha mencionado anteriormente, a la distancia geográfica y a la dificultad lingüística para acceder a estos espacios, por lo que podemos constatar que el trabajo de campo directo es relativamente reciente, quizá poco más de una década. Si se traza sucintamente el camino de la construcción de lo marroquí como objeto de estudio concreto, podría partirse de la noción de Oriente asociada por lo general a China, Japón o a la India, y que poco a poco fue abriendo su foco a Medio Oriente, acuciado por el suceso de las Torres gemelas en 2001.

Posteriormente, en el momento del estallido de las "primaveras árabes” en 2010, se incorporaron rápidamente otras disciplinas sociales al estudio del norte de África que sacaron, de cierta manera, al mundo árabe de un estatismo y ruralidad que le parecían inherentes por los estudios antropológicos extranjeros. 
En este sentido, pueden destacarse los trabajos y tesis desde las ciencias políticas de Indira Sánchez Bernal, enfocados en el sistema político marroquí (Sánchez, 2004 y 2012); la de Claudia Barona, actual profesora de relaciones internacionales y el politólogo Román López Villicaña, ambos referentes al fenómeno saharaui (Barona, 2004 y Villicaña, 2013) o los de la arqueóloga Azul Ramírez, centrados en la zona del Rif (Ramírez, 2013). Todos ellos comparten la característica de haber sido fruto del trabajo sobre el terreno, algo que estuvo vedado durante mucho tiempo para los estudiantes que debían conformarse con leer los textos teóricos a los que tenían acceso. A lo anterior hay que añadir el papel de España no solo desde el punto de vista logístico, ya que la ruta que siguen la mayoría de los investigadores es México-España-Marruecos, sino también en términos intelectuales puesto que es inevitable que algunos de los aspectos que se discuten, sobre todo en términos de política, estén permeados a su vez por las relaciones entre España y Marruecos que no han sido del todo fáciles.

Los trabajos de campo antes mencionados, a grandes rasgos han abarcado tres grandes zonas del país: el Rif, el centro y el Sáhara. Es importante señalar que, sobre todo Ramírez, ha hecho hincapié en la heterogeneidad del país que, durante mucho tiempo, se catalogó como un país árabe, incluso en los medios académicos. Su labor se extendió a la inclusión de un seminario de lengua y cultura amazigh coordinado junto al poeta rifeño Ouajd Karkar en la Escuela Nacional de Antropología e Historia. Este punto es fundamental porque representa uno de los fenómenos más importantes no solamente en Marruecos, sino en otros países del Magreb como Argelia.

La falta de reconocimiento de la especificidad amazigh al interior del país, fue durante muchos años una de las causas de los movimientos de reivindicación, lo que llevó a que en el año 2011 se reconociera en el artículo 5 de la Constitución marroquí el amazigh como lengua oficial del Estado. De la misma manera, respecto a la cuestión identitaria, se hizo constar en el preámbulo que su unidad "forgée par la convergence de ses composantes ara- 
bo-islamique, amazighe et saharo-hassanie, s'est nourrie et enrichie de ses affluents africain, andalou, hébräqque et méditerranéen" (La Constitution, 2011).

Respecto a otras investigaciones que se llevaron a cabo en el norte de México relacionados con Marruecos, estas privilegiaron la cuestión migratoria. Fueron llevadas a cabo en el Colegio de la Frontera Norte (COLEF) bajo títulos como "La condición femenina en las regiones fronterizas: los casos de México-Estados Unidos y Marruecos-España” en el 2013, coordinado por el granadino Antonio Trinidad, además de algunas publicaciones al respecto (Ribas, 2011 y Barajas \& Solís, 2015).

La posición geográfica de Marruecos y México conduce a que se pueda establecer, en ese rubro, otro paralelismo interesante, pues ambos son países tanto de tránsito como de recepción de inmigrantes, en el primer caso subsaharianos y en el segundo centroamericanos. Así se ha hecho constar en las diferentes ediciones del Foro Mundial sobre Migración y Desarrollo llevado a cabo desde 2006 por iniciativa de la Organización de las Naciones Unidas, y en el que algunos de los académicos del COLEF y de la Universidad de Zacatecas han tomado lugar.

De la misma forma, investigadores como el marroquí Mohamed Berriane, han propuesto tomar como ejemplo algunos programas mexicanos, específicamente el 3X1 (Berriane \& Adhergal, 2011), es decir, la aportación del gobierno federal de un porcentaje de dinero por cada dólar de las remesas, y aplicarlo al caso marroquí, en el que estas representan el 6.2\% del PIB. Esta condición ha supuesto que jóvenes investigadores, inscritos en los cada vez más abundantes estudios de Máster sobre América Latina en Marruecos, elijan la cuestión migratoria comparativa como uno de los temas, como es el caso de Soraya Harouchi del Máster L'Amérique Latine, globalisation, interculturalité et défis au XXI siècle de la Universidad Mohamed V de Rabat, cuya disertación se tituló "Migración femenina e infantil: Marruecos y México como dos países de encuentro" (Harouchi, 2020).

Ahora bien, consideramos que mientras en el medio académico se fue definiendo cada vez con más precisión lo marroquí 
como objeto y sujeto de estudio, no así en la mirada popular que lo siguió diluyendo no solo dentro de lo árabe, sino dentro de lo indio, como podemos constatar en algunas ferias de artesanías que los mezclan. Aunado a ello, contamos con otro elemento que ha jugado un fuerte papel en ambas direcciones: el cine y la televisión. Más allá del baisano Jalil, protagonizado por Joaquín Pardavé y donde cabían todos los árabes posibles, caracterizados siempre en función de lo económico, son las telenovelas las que han reforzado un estereotipo.

En el caso de México, un estereotipo negativo hacia lo marroquí solo compensa la valoración de sus productos estéticos como el aceite de argán. Así sucedió con la producción El Clon, de origen brasileño y adaptada para el público hispanohablante en 2010. En ella, presentaba la imagen de un marroquí con raíces religiosas bastante arraigadas que se oponía a un sentimiento tan popularmente defendible como el amor. Fue quizás uno de los primeros pasos en la toma de conciencia por parte del imaginario popular mexicano de "lo marroquí", puesto que además la telenovela estaba rodada en parte en la ciudad marroquí de Fez, lo que permitía hacerse una idea del paisaje.

En sentido inverso, no puede olvidarse el fuerte impacto de telenovelas como Guadalupe (1994) protagonizada por la actriz mexicana Adela Noriega, quien visitó Marruecos en ese mismo año debido a la fuerte popularidad de la producción televisiva que fue doblada al árabe, por lo que muchas amas de casa llegaron a pensar que ese era el idioma hablado en México. Una noticia recogida por Abdelhak Hiri del diario marroquí Al-Ittihad Al-Ichtiraki en el 2002, titulada "Las telenovelas mexicanas o la lengua diaria" afirmaba que: "Una parte considerable de la población logra aprender el árabe clásico a través de la adicción a las telenovelas mexicanas dobladas, después de haber sido una lengua reservada a los intelectuales y a los escolarizados" (Hiri, 2020, p. 244).

Al respecto, también de las telenovelas hay una anécdota de hace unos años cuando su proyección estuvo a punto de provocar un problema diplomático entre ambos países, ya que el ministro de Comunicación Marroquí, Mohamed el Khalfi, perteneciente 
al Partido Justicia y Desarrollo, de corte conservador, declaró en el 2014 que no quería que Marruecos se convirtiera en "el burdel de México" (Yabiladi, 2014).

De regreso al campo académico, puede constatarse que las nociones generales que se tenían sobre México en un principio, y que se mezclaban entre autores conocidos como Octavio Paz o pintoras como Frida Khalo - Marruecos no escapó a la fridomania de los últimos años - y personajes de telenovelas como Guadalupe, el Chavo del Ocho o Cantinflas, estaban igualmente permeadas por las noticias que de México se difunden a través de la prensa marroquí y que han sido minuciosamente estudiadas por Hiri, quien sitúa a México, después de Colombia, como el segundo país en importancia mediática en el país magrebí (Hiri, 2021). Este ordena, por importancia, los siguientes temas: cultura y sociedad (53\%), política (15\%), catástrofes naturales (12\%), relaciones Marruecos-México (11\%) y drogas y narcotráfico (9\%) (Hiri, 2020), lo que a grandes rasgos coincide con las noticias difundidas de América Latina, estudiadas también por Hiri en ocho periódicos de 1990 al 2015, donde cultura y sociedad ocupa también el primer sitio (17,8\%), seguido de las relaciones latinoamericanas con Marruecos (12,31\%), la política (11,19\%), la violencia $(6,21 \%)$, las guerrillas (5,55\%), las drogas y el narcotráfico (4,65\%) o la economía (3,33\%), por situar los más importantes (Hiri, 2021).

\section{Una mirada global hacia algunos ejemplos de diplomacia cultural de Marruecos con México, Panamá, Chile, Colombia y Argentina}

Tras mostrar brevemente algunos aspectos en la construcción especular de lo marroquí como objeto de estudio en México y de la imagen de México en Marruecos en las últimas décadas, se hará un repaso general sobre algunos vínculos académicos y culturales recientes entre Marruecos y algunos países de América Latina, los cuales resultan esperanzadores si se considera que se esfuerzan por mantener la línea horizontal de las relaciones Sur-Sur. Antes de comenzar, nos gustaría introducir un concepto que encontra- 
mos presente en algunas de las manifestaciones académicas y culturales: la diplomacia cultural.

Entendida esta en su acepción más común como "el intercambio de ideas, información, arte y otros aspectos de la cultura entre las naciones y sus pueblos para fomentar el entendimiento mutuo" (Cummings, 2003, p.1), su desempeño se ha asociado normalmente a la figura del Estado y sus instituciones. En el contexto de las relaciones entre Marruecos y América Latina, esta noción se ve fuertemente impulsada, según Bousseslam el Ouarrad, en los años ochenta ya que:

Marruecos definió un acercamiento más sistemático y estable con América Latina debido a la necesidad de constituir y mantener una plataforma de apoyo en torno a la cuestión del Sáhara. En aquel entonces, Marruecos duplicó sus acciones diplomáticas en varias naciones de la región como México, Chile, Perú y Argentina (El Ouarrad, 2021, p. 37).

El Ouarrad, cita justamente el discurso del Embajador Mohammed Ayachi, presentado al principio de este trabajo, donde el pasado andalusí es uno de los pilares fundamentales de dicha diplomacia cultural (El Ouarrad, 2021). Se observan entonces dos dimensiones de estas relaciones que están comprendidas en la actividad diplomática y en la cultural, y que de alguna manera se unen y/o se cruzan. Mencionaremos a continuación algunos ejemplos de estas que se han llevado a cabo en los últimos años y que expresan la necesidad de reforzar el vínculo entre ambas geografías.

Es importante subrayar, además, que gran parte de las actividades que mencionaremos enseguida, iniciaron o tuvieron continuidad durante la crisis sanitaria, como es el caso de las reuniones virtuales promovidas por Reyna Carretero, Mehdi Mesmoudi, Bachir Edkhil, Lamia el Amrani, Aziz Amahjour, Abdelhalak Najmi, Juan José Vagni, Diego Melo o Gloria Young, entre otros. 
100 Relaciones especulares entre Marruecos y América Latina: DE LA generación Beat a LA DIPLOMACIA CUlTURAL / Sandra Rojo Flores

\section{México}

México cuenta como antecedente con los ciclos de cine marroquí y conferencias llevadas a cabo con el apoyo de la representación diplomática marroquí en la Semana Árabe de México en los años 2011, 2012 y 2013. Este evento fue organizado por el Colegio de México y el Centro de Investigaciones en Docencia Económica bajo la dirección de la antropóloga mexicana Camila Pastor de María y Campos. En sentido inverso, destacamos la invitación de actores y directores mexicanos a festivales de cine marroquíes, sobre todo al norte del país. Es el caso del Festival Internacional de Cine y Memoria Común de Nador que dedicó su novena edición (2020) a las relaciones entre Marruecos y América Latina, y que incluía un ciclo de conferencias al respecto, como la del profesor Aziz Amahjour, que desde el año 2009 realiza un acercamiento al cine y cultura mexicana, reflejado en su libro sobre el cuento folklórico en México y el Mediterráneo (Amahjour, 2009).

Es igualmente importante la firma del convenio en el 2017 entre la Universidad Mohammed V de Rabat y la Universidad Nacional Autónoma de México, de las cátedras Graciela Hierro y Fátima Mernissi, ambas reconocidas académicas feministas. De esta última, ha sido designada recientemente como coordinadora la investigadora Reyna Carretero Rangel del Centro Regional de Investigaciones Multidisciplinarias de la UNAM. Carretero cuenta, además, con una reciente publicación que relaciona a México con Marruecos (Carretero, 2021) y colabora activamente con el Instituto de Estudios Saharianos Al-Andalus (Marruecos), dirigido por Bachir Edkhil.

De la misma manera, la labor del tangerino Mehdi Mesmoudi, desde la Universidad Autónoma de Baja California Sur es esencial para conocer la actualidad académica de las relaciones entre ambos países. Basta acercarse a las actividades del Ciclo de conferencias Marruecos-América Latina inauguradas en el 2018, así como al seminario "Marruecos y América Latina en la cartografía transhispánica” que coordina Mesmoudi. En la misma línea, los trabajos de la poeta tetuaní Lamia el Amrani en la Universidad 
Autónoma de Yucatán son representativos, pues esta ha servido como puente para que se lleven a cabo una serie de encuentros, además de formar parte de la publicación de poesía marroquí ofrecida por La Jornada Semanal denominada "El Marruecos de ellas: siete poetas contemporáneas" en el año 2014. Esta fue promovida por el poeta mexicano Jorge Valdéz Díaz-Vélez, quien fuera consejero Cultural de la Embajada de México en Marruecos. En esa misma línea de la poesía, es importante referirse a una de las últimas publicaciones al respecto, la de la poeta mexicana Ruth Pérez Aguirre y su compilación denominada "Mujeres que no callan. Marruecos” (Perez, 2021).

\section{Panamá}

En el caso de Panamá, es destacable el trabajo de la exembajadora y escritora panameña Gloria Young, quien junto a la investigadora Rajae Dakir, lideró un proyecto conjunto entre la Universidad de Panamá y la Universidad Hassan II de Casablanca, denominado "Historia de Marruecos y Panamá en la voz poética de sus mujeres” (Young, 2018). Young, quien lidera la página de Facebook Cooperación Sur-Sur: Panamá y Marruecos, forma parte activa de las reuniones llevadas a cabo dentro del programa de cooperación Ibn Khaldoun coordinado por Abdelmouneim Bounou, de la Universidad Mohamed V de Rabat, como "20 años de relaciones entre Marruecos y América del Sur I y II" realizadas de forma virtual en marzo y abril del 2021, o la última de ellas "Las relaciones diplomáticas entre Marruecos y América Latina en tiempos de pandemia", también en formato virtual, celebrada el 16 de junio.

El proyecto central del programa de cooperación se denomina "Las relaciones diplomáticas entre Marruecos y América Latina: estado de la situación y perspectivas de cooperación" y el profesor Bounou coordina, además, el programa de Máster L'Amérique Latine globalisation interculturalité et défis au XXI siècle, de esa misma Universidad. 
102 Relaciones especulares entre Marruecos y América Latina: De la generación Beat a LA Diplomacia CUltural / Sandra Rojo Flores

\section{Chile}

Chile representa un caso especial por el ejercicio como embajador del poeta marroquí Abdelkader Chaui de 2009 a 2016. En primer lugar, se trata de un antiguo preso político en el contexto de los años de plomo de los que hablamos en líneas precedentes, cuyas ideas le hicieron pasar 16 años en prisión. Por otro lado, su actividad poética le llevó a impulsar un reconocido intercambio cultural entre ambos países, y algunos de sus trabajos han sido publicados en español. A ello, se suman las actividades del Centro Mohammed V para el diálogo de civilizaciones, inaugurado en mayo de 2007 en Coquimbo y ejemplo claro de diplomacia cultural. De la misma manera, cuentan con iniciativas universitarias como la Cátedra Al Andalus-Magreb de la Facultad de Artes Liberales de la Universidad Adolfo Ibáñez dirigida por el historiador chileno Diego Melo, impulsor de varios proyectos entre ambos países (Melo, 2021) y las actividades virtuales tampoco han sido una excepción. La última de ellas, un webinar titulado "Diálogos interculturales Chile-Marruecos en sus sesenta años de relaciones diplomáticas”, organizado el 2 y 9 de junio de 2021.

\section{Argentina}

Una de las características de las relaciones entre Marruecos y Argentina tiene que ver con la historia de la emigración árabe a estos países abordada por historiadores marroquíes como Abdeluahed Akmir (2011) o la socióloga argentina Diana Epstein (2011), ya que en la segunda mitad del siglo XIX empezaron a llegar judíos sefardíes de origen marroquí a este país, lo que constituye una particularidad respecto al resto de América Latina. En la actualidad, y en la línea de los recientes proyectos bilaterales en materia de cultura, debemos nombrar, desde el ámbito de las Relaciones Internacionales, a investigadores como Juan José Vagni, quien ha dedicado gran parte de su trabajo a las relaciones entre Marruecos y América Latina y es un invitado imprescindible en los Foros donde se discute esta cuestión. Fue el caso del Congreso Internacional "Mundo Árabe-América Latina y el Caribe: una dinámica 
renovada. Marruecos, puente con América Latina y puerta hacia África”, organizado en Rabat por el Instituto de Estudios Hispano Lusos y el Consejo para las Relaciones Árabes con Latinoamérica y el Caribe, en el año 2018. Vagni coordina, además, el grupo de trabajo de CLACSO Medio Oriente y norte de África, donde se dan cita gran parte de los investigadores relacionados con Marruecos. Finalmente, no puede pasar desapercibido el poeta cordobés Leandro Manuel Calle, quien, además de ser traductor al español de poetas como Abdelatif Laâbi, es el director de la Colección Biblioteca Marroquí de la editorial Alción, que publica en español a autores marroquíes como Abdelkader Chaoui, Siham Boulal, Mohamed Lahchiri, Ouidad Benmoussa, Tahar Benjelloun, Rachid Benzine o Driss Chaibi. Esta labor resulta fundamental para poder acercar parte de la producción literaria marroquí al público hispano, y sobre todo latinoamericano.

El camino que se presenta parece bastante fructífero, sobre todo en los tiempos en los que se aboga por una "descolonización" del conocimiento, como bien lo nombra el sociólogo portugués Boaventura de Sousa Santos (Santos, 2010).

\section{Reflexiones finales}

En los párrafos anteriores, se han mostrado algunos paralelismos y relaciones especulares entre Marruecos y América Latina, en especial México. Lo que en un primer momento podría presentarse como un objeto de estudio tradicional para disciplinas como la antropología, se ha convertido en un sujeto con el cual dialogar y compartir problemáticas como las derivadas de la vulnerabilidad socioeconómica producto del colonialismo.

Desde esa premisa, es decir, la posibilidad de establecer un análisis comparativo y no vertical como se lleva a cabo desde la academia española o francesa se ha presentado una serie de momentos históricos que van desde los años cincuenta hasta la actualidad, poniendo en relación con Marruecos con América Latina, en particular con México. Como se ha podido observar, por su estructura socioeconómica y cultural, ambos sirvieron como 
escenario para el mismo grupo de autores que se consideraban contraculturales y que jamás apreciaron, según los testimonios de la época, a la población local marroquí y mexicana.

De distinta índole, una contracultura vio la luz en Marruecos durante los “años de plomo", período políticamente dramático en el que muchos activistas e intelectuales fueron desaparecidos y encarcelados, y cuyas obras literarias y artísticas fueron prácticamente desconocidas para el mundo hispanohablante. Muchos de ellos intentaron establecer un paralelismo con la situación de América Latina que en ese momento pasaba por una situación política similar.

Posteriormente, se recurrió de nuevo a países como Chile o Argentina para tomar referencias que pudieran servir en el proceso de reconciliación nacional marroquí. Ambos ejemplos sirvieron de precedente para reflexionar sobre la manera en que lo marroquí como objeto de estudio e imaginario se había construido en México, siendo esto una herencia de la academia europea y estadounidense, y habiendo importado los mismos prejuicios, mantenidos por la dificultad de realizar trabajo de campo directo. Fue este el que permitió empezar a definir un Marruecos menos arcaico y homogéneo para introducir, gracias a algunas investigaciones, elementos como su carácter pluricultural. Entonces se empezaron a gestar gracias a la diplomacia cultural una serie de encuentros y proyectos que se mantuvieron o dieron lugar a otros nuevos gracias a la posibilidad de utilizar las plataformas virtuales lo que, consideramos, es un paso importante para el mantenimiento y el fortalecimiento de las relaciones Sur-Sur.

\section{Referencias}

Akmir, A. (2011). Los árabes en Argentina. UNR Editora. Amahjour, A. (2000). El cuento folklórico en México y en la Cuenca del Mediterráneo. Estudio semiótico de textos pertenecientes a las dos tradiciones. Ediciones de la Fundación Universitaria Española. Ayachi, M. (1992). La política exterior de Marruecos. Colombia Internacional, 20, 17-24. 
Barajas, E. \& Solis, M. (2015). Fronteras comparadas: desarrollo, trabajo y migración México-Estados Unidos/Marruecos-Unión Europea. COLEF.

Barona, C. (2004). Hijos de la nube. Cuadernos de Langre.

Bartra, R. (2017). Memorias de la contracultura. Letras libres, 105. https://www.letraslibres.com/mexico/memorias-la-contracultura

Berriane, M. \& Adhergal, M. (2011). Migration/développement Etude de faisabilité pour l'adaptation du programme mexicain 3 pour 1 au cas du Maroc. GTZ Migration and Development. Bukowski, Ch. (1998). Escritos de un viejo indecente. Anagrama. Burroughs, W. (1985). Queer. Viking Press.

Burroughs, W. (1989). El almuerzo desnudo. Anagrama.

Burroughs, W. (2006). Marica. Anagrama.

Casielles, L. (2018). Los cantos inolvidables:Souffles, una revista marroqui de poesía y politica entre el colonialismo y los años de plomo. Alción.

Carretero, R. (2019). Dolce convivio. Composibles del trashumante urbis. CRIM-UNAM.

Choukri, M. (2012). Paul Bowles, el recluso de Tánger. Cabaret Voltaire.

Choukri, M. (2014). El pan a secas. Cabaret Voltaire.

Cummings, M. (2003). Cultural Diplomacy and the United States Government: A Survey. Center for Arts and Culture.

Derkaoui, M. (dir.). (1976). De quelques événements sans signification [película]. Documental, 76'.

Geertz, C. (1994). Islam Observado: Desarrollo Religioso en Marruecos e Indonesia. Prensa Ibérica.

El Ouadi, S. (2001). Le marié. Candide au pays de la torture. Méditerranée.

El Ouarrad, B. (2021). En torno a la diplomacia cultural entre España, América Latina y Marruecos. En D. Melo (ed.), África, Iberoamérica y Europa: intercambios culturales desde una perspectiva multidisciplinar. (pp.27-43), Dykinson.

Epstein, D. (2011). Marroquíes de origen judío en Argentina. Cohesión y dispersión comunitaria. Revista de Historia. 12, 
57-69, http://revele.uncoma.edu.ar/htdoc/revele/index.php/ historia/article/view/81/79

García Linares, R. J. (2020). El islam en el norte de México: los roles de género en la construcción y negociación de la identidad religiosa de las mujeres y de los hombres del Centro Islámico del Norte en Monterrey [Tesis de doctorado, Universidad Nacional Autónoma de México].

García-Robles, J. (2014). La bala perdida. William Burroughs en México. Editorial La Moderna.

García-Robles, J. (2018). Kerouac. La generación beat. Juan Pablos Editor.

Gellner, E. (1969). Sains of the Atlas. University of Chicago Press. Goytisolo, J. (2014, 05 de julio). Tánger, Burroughs y la beat generation. El Pais. https:/elpais.com/elpais/2014/07/01/opinion/1404210763_817107.html

Harouchi, S. (2020). Migración femenina e infantil: Marruecos y México como dos paises de encuentro. [Tesis de maestría, Universidad Mohamed V].

Hart, M. (1976). The AithWaryaghar of the Moroccan Rif. University of Arizona Press.

Hiernaux-Nicola, D. (2007). México, el espacio mágico y efímero de los beats. Revista Casa del Tiempo, 100, 32-41.

Hiri, A. (2020). Presencia e imagen de México en el periódico marroquí Al-Ittihad Al-Ichtiraki. RAEIC, Revista de la Asociación Española de Investigación de la Comunicación. 7(14), 236-257.

Hiri, A. (2021). Tratamiento informativo de América Latina en la prensa marroquí. En D. Melo (Ed.) África, Iberoamérica y Europa: intercambios culturales desde una perspectiva multidisciplinar. (pp. 163-182). Dykinson.

Idrissi, T (dir). (2014). Rif 58-59. Briser le silence [Película]. Farfira Films 76'.

Kerouac, J. (1960). Tristessa. Avons Publications.

Lewis, O. (1965). Los hijos de Sánchez. Autobiografía de una familia mexicana. Fondo de Cultura Económica. 
Llorente, M. (2014, 22 de noviembre). Mohamed Mrabet: «Paul Bowles fue un hijo de la gran puta. No me pagó ni un franco". El Mundo https://www.elmundo.es/cultura/literatura/2019/11/22/5dd6d9cffdddffbe2e8b4585.html

La Constitution (2011). Royaume du Maroc: Secrétariat Général du Gouvernement.

http://www.sgg.gov.ma/Portals/0/constitution/constitution_2011_Fr.pdf

Macías, S. (2000). Marruecos en la literatura latinoamericana. Ministerio de Educación.

Maraini, T. (1990). Écrits sur l'art, Al Kalam Éditions.

Martínez Elizondo, I. (1884). Recuerdos de un viaje en América, Europa y África. Librería de P. Brégi.

Martínez Elizondo, I. (1886). Viaje universal. Visita a las cinco partes del mundo. José S. Molins.

Mdidech, J. (2002). La chambre noir. Eddif Maroc.

Melo, D. (2021). África, Iberoamérica y Europa. Intercambios culturales desde una perspectiva multidisciplinar. Editorial Dykinson.

Mendoza, J. (2014, 22 de noviembre). Tánger, patria emocional y generación beat. Diario El País.

https://www.elmundo.es/cultura/2014/11/22/546fa0d822601dc1la8b4574.html

Merzouki, A. (2000). Tazmamart Cellule 1010. Méditerranée/ Tarik Éditions.

Pérez, R. (ed.). (2021). Mujeres que no callan. Marruecos. Ediciones htuRquesa Cartonera.

Rabinow, P. (1977). Reflexiones sobre un trabajo de campo en Marruecos. Júcar Universidad.

Ramírez, A. (2013). Los santos (IMRABDEN) y el paisaje cultural entre los AYT ALI U AISSA (AYT ILI U IISSA) del Rif central marroqui [Tesis de Doctorado, Escuela Nacional de Antropología e Historia].

Ribas, N. (2011). El Río Bravo Mediterráneo. Las regiones fronterizas en la época de la globalización. Bellaterra. 
Sánchez Bernal, I., (2004). Un mito útil en la seguridad nacional marroquí: las reivindicaciones territoriales sobre la RASD [Tesis de maestría, El Colegio de México].

Sánchez Bernal, I. (2012). El sistema político marroquí en la encrucijada del cambio político 1997-2007: monarquía, partidos políticos, movimientos islamistas, sociedad civil y organización civil. [Tesis de doctorado, El Colegio de México].

Sefrioui, K. (2013). La revue Souffles 1966-1973: espoirs de révolution culturelle au Maroc. Éditions du Siroco.

Soussa, B. (2010). Descolonizar el saber, reinventar el poder. Ediciones Trilce.

Taboada, H. (1998). Un orientalismo periférico. Viajeros latinoamericanos 1786-1920. Estudios de Asia y África, XXXIII(2), 285-305.

Taboada, H. (2003). Nuestra América y el islam. La mirada en el tercer milenio. Archipiélago. Revista cultural de Nuestra América, 9(40), 11-14.

Vagni, J.J. (2016). América como modelo para la actuación de España en el Protectorado en Marruecos. La visión de Rodolfo Gil Benumeya. TRANSMODERNITY: Journal of Peripheral Cultural Production of the Luso-Hispanic World., 35-53.

Vagni, J.J. (2016-2017). De la hermandad hispano árabe a la diplomacia cultural: la construcción del interés de Marruecos por América Latina bajo mediación española. Norba. Revista de Historia. 29-30, 239-258.

Villicaña, R. (2013). El problema del Sahara occidental: una perspectiva geopolitica. EDAF.

Wittfogel, K. (1966). Despotismo oriental: estudio comparativo del poder totalitario. Guadarrama.

Zekri, K. (2011). Écrire le carcéral: histoire, mémoire et écriture de soi. Expressions maghrébines, 10(2), 7-32.

Yabiladi. (2014, 07 de junio). Le feuilleton mexicain de Mustapha ElKhalfi risque de tourner au vaudeville [Billet Fabrique]. https://www.yabiladi.com/articles/details/26601/feuilleton-mexicain-mustapha-khalfi-risque.html

Young, G. (2018). Historia de Marruecos y Panamá en la voz poética de sus mujeres. Publicaciones del Laboratorio de Investigación. 